\title{
EL CENTRE BELGE DE PÉDAGOGIE DE LA MATHÉMATIQUE (1958-1973): nota histórica
}

\author{
O centro pedagógico Belga de matemática (1958-1973): \\ nota histórica
}

\section{Modesto Sierra Vázquez}

Profesor Titular de Universidad Facultad de Educación. Universidad de Salamanca, Espanha. Salamanca, ES - Espanha, e-mail: mosiva@usal.es

\section{Resumen}

En este trabajo se presenta de manera resumida el desarrollo histórico del Centre Belge de Pédagogie de la Mathemátique (19581973), creado por Georges Papy, haciendo especial hincapié en el desarrollo curricular que abarca desde los 6 a los 18 años; la influencia de la obra de Papy en el Movimiento de la Matemática Moderna está ampliamente reconocida; se analizan los fundamentos de la reforma y se muestran algunos aspectos críticos de la misma.

Palabras-clave: Movimiento de la Matemática Moderna; Centre Belge; Papy.

\section{Resumo}

Neste trabalho apresenta-se, de forma resumida, o desenvolvimento histórico do Centro Belge de Pédagogie da la Mathemátique (19581973), criado por Georges Papy, dando especial ênfase ao desenvolvimento curricular entre os 6 aos 18 anos; a influência 
da obra de Papy no Movimento da Matemática Moderna está amplamente reconhecida; analisam-se os fundamentos da reforma e põem-se em evidência alguns aspectos críticos da mesma.

Palavras-chave: Movimento da Matemática Moderna; Centro Belge; Papy.

\section{INTRODUCCIÓN}

La reforma de la enseñanza de las Matemáticas llevada a cabo en los años sesenta es un hecho notable en la historia de la educación matemática ya que originó un vasto movimiento que pretendió, entre otros fines, salvar la enorme distancia que se había creado entre la Matemática como ciencia y la que se enseñaba en el secundario. En este contexto se inscribe la investigación que llevé a cabo (SIERRA, 1989) sobre el Centre Belge de Pédagogie de la Mathématique, en adelante CBPM, institución que aglutinó en torno a Georges Papy y su mujer Fréderique a una serie de matemáticos, pedagogos, y profesores de Matemáticas, que realizaron una intensa acción innovadora entre los años 1958 y 1973. La influencia del Centro se extendió a todo el mundo occidental como lo prueba el hecho de que ciertas obras de su promotor hayan sido traducidas a catorce lenguas y que miembros del CBPM diesen cursos y conferencias en unos cincuenta países; además, a lo largo de esos años el CBPM acogió en su seno a investigadores de una veintena de naciones que al regresar a sus países de origen difundieron su metodología.

Las fuentes utilizadas para la investigación se pueden clasificar en primarias y secundarias. Las primarias corresponden a publicaciones originales del CBPM y las secundarias a documentos de autores que han escrito sobre esta institución. Se han revisado 121 publicaciones de G. Papy y 44 de F. Papy.

El CBPM creó la revista NICO, publicación periódica donde se exponían las experiencias realizadas; NICO es el diminutivo de Nicolás Bourbaki, lo que es una prueba más en la influencia que este colectivo de matemáticos ejerció sobre la reforma emprendida. Pues bien, en la investigación se ha realizado un vaciado bibliográfico de los números de esta revista.

Esta presentación se ha organizado del siguiente modo:

- antecedentes;

- desarrollo histórico del Centro;

- conceptuación previa de la Matemática;

Rev. Diálogo Educ., Curitiba, v. 8, n. 25, p. 633-645, set./dez. 2008 
- reforma de los métodos de enseñanza;

- desarrollo curricular;

- análisis crítico de ciertos aspectos de la reforma.

Insisto en que se trata de una presentación resumida con el único objetivo de mostrar las líneas generales de la reforma emprendida por G. Papy y sus colaboradores del CBPM.

\section{Antecedentes: actividad internacional en la enseñanza de las Matemáticas}

Se han identificado aquellas tendencias en educación que de modo implícito han influido en la filosofía del CBPM; estas tendencias tenían la idea común de una democratización de la enseñanza, con una escuela "para todos", concebida como institución transformadora. En este sentido en Europa aparecen desde principios de siglo las "Escuelas Nuevas", la llamada Pedagogía "científica" con las figuras de María Montessori (1870-1952) y O. Decroly (1871-1932); en los Estados Unidos sobresale la figura de Dewey (1859-1952). Todo ello provocó un clima cultural en Europa creando presiones específicas a favor del cambio.

Asimismo, se ha estudiado la actividad internacional de enseñanza de las Matemáticas en el periodo 1900-1960; se puede asegurar (HOWSON, 1979; HOWSON; KEITEL; KILPATRICK, 1981; CASTELNUOVO, 1963) que desde comienzos de siglo hay una gran preocupación entre los educadores matemáticos que cristaliza en la fundación de asociaciones, publicación de revistas y celebración de congresos y reuniones. Después de la Segunda Guerra Mundial se crea la Commission Internationale pour l'Etude et la Amelioration de l'Enseignement des Mathématiques (CIEAEM) fundada por el matemático G. Choquet, el psicólogo J. Piaget y el pedagogo C. Gattegno; el mérito de la CIEAEM en esos años fue insistir en la práctica de la enseñanza, atacando efectivamente los problemas pedagógicos que generaba dicha práctica.

Sin embargo, el verdadero golpe maestro para iniciar una reforma en profundidad no proviene de ningún Ministerio de Educación, sino de una organización económica: la Organisation Européenne de Coopération Economique (OECE) que en 1963 toma su nombre actual de Organisation de Coopération et de Devéloppement Economique (OCDE). Auspiciado por la OECE se celebra en 1959 el Coloquio de Royaumont (Francia) seguido del Seminario de Dubrownite (Yugoslavia) en el verano de 1960 donde se establecieron las líneas maestras y los programas para la enseñanza secundaria. Precisamente, G. Papy y sus colaboradores del CBPM desarrollaron, a nivel teórico y en el aula, las ideas,

Rev. Diálogo Educ., Curitiba, v. 8, n. 25, p. 633-645, set./dez. 2008 
programas y líneas metodológicas establecidas en el Coloquio; y posteriormente extendieron las mismas a la enseñanza primaria, a iniciativa de M.H. Stone, presidente del Coloquio de Royaumont.

\section{Desarrollo histórico de la reforma del CBPM}

En el desarrollo histórico el Centro se distinguen cuatro periodos perfectamente diferenciados.

- Primer periodo 1958-1961: Inicio de la reforma por Fréderique Lenger (más tarde esposa de G. Papy) y W. Servais en ciertas clases de escuela Normal, Sección de preescolar. El Profesor de la Universidad de Bruselas George Papy se incorpora a la experiencia a requerimiento de sus promotores.

El 24 de Mayo de 1961 se crea jurídicamente el CBPM, como Asociación privada, sin ánimo de lucro; el artículo 1 de sus estatutos establece que la Asociación tiene por objeto el estudio y la mejora de la enseñanza de las Matemáticas y que, en particular contribuirá a la promoción, desarrollo y difusión de la enseñanza de la Matemática moderna:

- Segundo periodo 1961-1964: se lleva a cabo la reforma en el secundario inferior (alumnos 12-15 años);

- Tercer periodo 1964-1967: reforma del secundario superior, sección científica (alumnos 15-18 años);

- Cuarto periodo 1967-1973: la reforma se extiende a la enseñanza primaria (alumnos 6-12 años).

En cada uno de estos periodos se ha estudiado: desarrollo curricular, experiencias realizadas, publicaciones, formación continua del profesorado, relación con las instituciones educativas y proyección internacional del Centro.

\section{Las matemáticas en la reforma}

Una de las ideas motrices de la reforma es la separación cada vez más grande entre la Matemática como ciencia viva y la que se enseñaba en el secundario. No es posible comprender el alcance de la renovación llevada a cabo por el CBPM si no se tiene una idea precisa de la evolución de las Matemáticas desde por lo menos mediados del siglo pasado hasta los años cincuenta en que comienza la reforma. De esta historia de la Matemática he considerado cuatro

Rev. Diálogo Educ., Curitiba, v. 8, n. 25, p. 633-645, set./dez. 2008 
aspectos por ser precisamente los señalados por G, Papy. Pero al recurrir a la historia lo hacen con el fin de justificar mediante ella las reformas emprendidas, es decir, plantean la historia al servicio de una idea determinada.

Estos aspectos son:

- Fundamentos de la Geometría: como es bien conocido, Hilbert (1899) en Grundlagen der Geometrie estableció un sistema de axiomas lógicamente satisfactorio. Artin (1957) en su obra Geometric Algebra recoge algunos de los progresos realizados desde 1900; estas dos obras no pueden constituir de ninguna manera manuales de enseñanza secundaria, pero han sido fuente de inspiración de G. Papy en la reforma emprendida.

- Aritmetización del Análisis: en la segunda mitad del siglo XIX se fundamenta el Análisis matemático con el proceso conocido como "aritmetización del Análisis" con la construcción rigurosa del número real. Este proceso ha influido notablemente en la presentación del Análisis en la reforma de la enseñanza secundaria superior.

- Teoría de conjuntos: constituye el nuevo cuadro unitario en el que Papy y colaboradores del CBPM cimentan la reforma.

- Estructuras y método axiomático: frente a la disparidad de teorías matemáticas que amenazaban convertir a esta ciencia en un pulpo de infinitos tentáculos, aparece el concepto de "estructura"; bajo la diversidad de métodos de las diversas disciplinas hay un método común, el "método axiomático". El representante más caracterizado de esta tendencia es el colectivo de matemáticos agrupados bajo el nombre de Nicolás Bourbaki, espejo en el que se mira el CBPM, para fundamentar su reforma de enseñanza de las matemáticas.

G. Papy y colaboradores consideraban que así como los Elementos de Euclides desarrollaban la Matemática de su tiempo, la obra de N. Bourbaki contiene la matemática base del nuestro. Surgen así dos ideas claves en la reforma del CBPM:

La primera idea es la expresión "Matemática moderna"; "Matemática de nuestro tiempo".

Matemática moderna $=$ Matemática que usa conjuntos y grandes estructuras.

Matemática moderna $=$ Matemática que ha abandonado el estado artesanal para pasar a la era industrial.

Rev. Diálogo Educ., Curitiba, v. 8, n. 25, p. 633-645, set./dez. 2008 
En la conceptuación de Papy sus colaboradores del CBPM, las estructuras-madre, según las define Bourbaki, serían análogas a las herramientas de la industria ya que permiten la economía de pensamiento y evitan la repetición de razonamientos; deben ser introducidas progresivamente según se va construyendo el edificio matemático, ya que presentadas al final cuando está ya construido no tiene ningún sentido. El hilo conductor de todo el proceso es el método axiomático; en la enseñanza del Centro se utilizará el método axiomático progresivo que consiste en que los axiomas no se dan desde el principio sino que se van introduciendo a lo largo de la teoría, de modo progresivo y motivado.

La segunda idea es Matemáticas "por todas partes". G. Papy afirma que durante mucho tiempo la Matemática ha sido señalada como elemento de cultura y de formación de la mente, pero que la situación ha cambiado profundamente al invadir todos los dominios del pensamiento racional; de ahí que haya que preparar a las nuevas generaciones en esta Matemática, para que puedan comprender el resto de las ciencias. Sin embargo, paradójicamente, a lo largo de la experiencia desarrollada no aparecen aplicaciones de la Matemática a otras ciencias.

\section{La reforma de los métodos de enseñanza}

La reforma emprendida por el CBPM no fue en modo alguno una simple reconsideración de los contenidos a la luz de la evolución de la Matemática como ciencia; fue también y sobre todo una reforma de los métodos de enseñanza. Modernización del contenido y renovación de los métodos de enseñanza van indisolublemente unidos.

En la reforma emprendida no hay explícita ninguna teoría del aprendizaje o de la instrucción y tampoco es su intento hacer ninguna formulación en esos términos. La fuente de inspiración de su reforma habría que buscarla en una reflexión sobre la pedagogía interna de las Matemáticas según la concepción epistemológica dominante en la época, con el posterior diseño de medios pedagógicos que se desarrollaban en el aula: actuando por el método error-acierto se iban afinando cada vez más esos medios pedagógicos pero siempre en interacción con los alumnos en las clases experimentales. Esta renovación de los métodos de enseñanza va a descansar, a mi juicio, sobre cuatro pilares:

- la idea de que los conceptos fundamentales de la Matemática de nuestro tiempo están en el conocimiento común;

- la necesidad de partir de situaciones pedagógicas adecuadas para refinar de modo progresivo ese conocimiento común hasta convertirlo en conocimiento matemático. Esta pedagogía de las situaciones va a estar directamente inspirada en las situaciones matemáticas de C. Gattegno;

Rev. Diálogo Educ., Curitiba, v. 8, n. 25, p. 633-645, set./dez. 2008 
- creación de medios pedagógicos esencialmente no verbales, como son:

Lenguaje de las cuerdas, que consiste en representar conjuntos mediante diagramas de Venn, coloreando las cuerdas de dichos diagramas con colores diferentes según el resultado de la operación.

Lenguaje de las flechas, que va a soportar el aspecto relacional de las Matemáticas. Este lenguaje se materializará en grafos sagitales multicolores, utilizando colores diferentes para expresar diversas relaciones que aparecen en una situación determinada; precisamente a esos grafos se les denomina "papygrmas" en honor de Papy.

Demostraciones por "bandas dibujadas", asemejándose a cintas de películas.

Convención verde-rojo, con la idea de presentar ciertas situaciones fundamentales de la primera enseñanza del Análisis.

Minicomputer de Papy

- pedagogía de la afectividad, con la creación de cuentos matemáticos.

\section{Desarrollo curricular}

Como ya se ha dicho, entre los años 1958 y 1973 se lleva a cabo la reforma en los distintos niveles de enseñanza.

Secundario inferior (alumnos 12-15 años)

En el secundario inferior, en la investigación realizada se han considerado cuatro grandes núcleos en torno a los que se puede vertebrar la reforma del CBPM destacando, sin lugar a dudas, la reconstrucción de la geometría afín y métrica del plano. Los otros tres núcleos son: conjuntos, relaciones y funciones, números naturales y enteros.

Ciñéndome a la geometría, hay que señalar que el objetivo de la reforma del CBPM en el secundario inferior es la reconstrucción del edificio geométrico hasta llegar a la estructura de $\pi_{0}$ como espacio vectorial euclídeo definido sobre el cuerpo de los números reales.

En cuanto a su curso temporal:

En la clase sexta (12-13 años), se introducen los axiomas de incidencia y orden; se trabaja con los conceptos relacionados con estas cuestiones y se estudian las traslaciones.

Rev. Diálogo Educ., Curitiba, v. 8, n. 25, p. 633-645, set./dez. 2008 
En la clase quinta (13-14 años), se establece la estructura del plano como espacio vectorial real realizándose simultáneamente dicha construcción y la del cuerpo de los números reales.

En la clase cuarta (14-15 años), se llega a la estructura de espacio vectorial euclídeo y se trabaja en dicho espacio vectorial.

En esta reconstrucción se van a emplear a la vez las nociones conjuntistas y el método axiomático progresivo.

Secundario superior, sección cientifica (alumnos 15-18 años)

Por lo que se refiere al secundario superior (sección científica) he considerado tres materias que se desarrollan a lo largo de los tres cursos: Álgebra lineal, Análisis y Aritmética.

En cuanto al Álgebra lineal, comprende:

- Espacios vectoriales de dimensión finita;

- Plano vectorial euclídeo;

- Geometría del espacio;

- Formas bilineales y cuadráticas.

La idea motriz esa que hay que enseñar los espacios vectoriales en el secundario por ser una de las estructuras fundamentales de nuestro tiempo. Siguiendo el camino ya marcado en el secundario inferior, continúa predominando el enfoque conjuntista y se sigue utilizando como medio pedagógico los papygramas y las demostraciones por bandas dibujadas (películas).

En Análisis, la distribución de contenidos, a grandes rasgos, es la siguiente:

- clase tercera (15-16 años): Números reales;

- clase segunda (16-17 años): Elementos de topología, continuidad de funciones, límite y derivadas;

- clase primera (17-18 años): Fórmula de Taylor, continuidad uniforme, integración, medida de ángulos y funciones circulares.

A mi juicio, los rasgos más destacados en la enseñanza del Análisis son:

- la influencia bourbakista y no solamente en el espíritu sino incluso en algunos aspectos locales donde se calca literalmente a Bourbaki;

- se linealiza la teoría poniendo en evidencia los espacios vectoriales que van apareciendo, con sus aplicaciones lineales;

- el lenguaje de los $\varepsilon$ y los $\delta$, desaparece de la exposición y sólo hace su entrada al tratar la continuidad uniforme, y esto es así porque en todo momento las definiciones de carácter topológico priman sobre las métricas;

Rev. Diálogo Educ., Curitiba, v. 8, n. 25, p. 633-645, set./dez. 2008 
- en ningún momento se exponen aplicaciones a otras ciências;

- la visión geométrica y el punto de vista conjuntista presiden toda la enseñanza del Análisis;

- en cuanto a los medios pedagógicos, se acentúa la convención verde-rojo;

La Aritmética comprende: Combinatoria. Aritmética de los números enteros, Anillos conmutativos y Cuerpos.

\section{Enseñanza primaria (alumnos 6-12 años)}

La reforma de la enseñanza primaria, se llevó a cabo empezando por primer curso (alumnos 6-7 años) y continuando hasta sexto curso (alumnos 11-12 años) inspirándose en la realizada en el secundario; de hecho numerosas cuestiones de enseñanza secundaria son trasladadas a la primaria con la clara intención de comenzar la enseñanza de los conceptos de la matemática de nuestro tiempo lo antes posible. La experiencia, conducida por Fréderique Papy, se desarrolló en la Escuela Normal Primaria Berkendael (Bruselas); durante los seis cursos Fréderique Papy trabaja con los mismos niños, alrededor de veintisiete, lo que hace posible seguir sus progresos, colaborando en la experiencia Asistentes del CBPM.

Los libros en los que se relata la reforma no son manuales escolares ni tampoco guías didácticas para el profesor, sino descripciones fieles y detalladas de las lecciones experimentadas; estas obras constituyen la serie Les enfants et la mathématique, habiéndose publicado únicamente los correspondientes a los cuatro primeros cursos. Un aspecto importante de mi investigación ha sido reconstruir el curso cronológico de las lecciones.

Las 610 lecciones impartidas se distribuyen del siguiente modo:

\begin{tabular}{lll}
\hline Temas & $\mathbf{N}^{\mathbf{0}}$ Lecciones & $\%$ \\
\hline Conjuntos y relaciones & 81 & 13,3 \\
Números y operaciones & 232 & 38 \\
Geometría & 132 & 21,6 \\
Matemática aplicada y Problemas & 83 & 13,6 \\
Razonamiento lógico y conjuntista & 39 & 6,4 \\
Estructuras & 43 & 7,1 \\
\hline Total & 610 & 100 \\
\hline
\end{tabular}

Rev. Diálogo Educ., Curitiba, v. 8, n. 25, p. 633-645, set./dez. 2008 
Algunas de las características de la metodología seguida son las siguientes:

- se continúa y acentúa la pedagogía de las situaciones didácticas; seleccionados los contenidos matemáticos, se crean situaciones que interesan a los niños provocando reacciones que conduzcan a esas nociones;

- es un desarrollo curricular en "espiral" en el sentido de que un mismo concepto es tratado desde puntos de vista diferentes a lo largo de los cuatro cursos;

- se utilizan los lenguajes no verbales ya descritos anteriormente;

- se llevan a cabo procesos de matematización que se hacen habitualmente sobre problemas abiertos, poniendo más énfasis en el aprendizaje que en la enseñanza;

- se crea un clima de afectividad hacia los niños.

Los resultados de la experiencia, según sus autores, revelan que después de una enseñanza adecuada en los tres primeros años de la enseñanza primaria es posible adquirir en el cuarto año lo que ellos llaman un segundo nivel de comprensión de la matemática. Este segundo nivel tiene un doble aspecto:

1 - Construcción del conjunto de los números reales mediante un largo camino axiomatizante que conducirá al axioma de continuidad.

Edificación casi completa del plano vectorial real.

Puesta en evidencia de ciertas nociones que serán esenciales para el acceso a un tercer nivel: grupo, propiedad distributiva, conjunto infinito.

2 - Tratamiento más sistemático de ciertos aspectos de la matemática aplicada.

Asimismo señalan que a lo largo de su experiencia no han encontrado los estadios de Piaget; sin embargo no explicitan los nuevos estadios encontrados ni es propósito de su investigación hacer una búsqueda de los mismos. Se limitan a afirmar que las experiencias realizadas deberían provocar nuevos estudios aplicando la técnica clínica de Piaget, para tratar de determinar la frontera entre lo que era el resultado de una enseñanza tradicional de las Matemáticas y el auténtico desarrollo intelectual del niño.

\section{Aspectos críticos de la reforma}

Una parte importante de esta investigación es el análisis crítico de la reforma llevada a cabo por el CBPM; este análisis debe realizarse, a mi juicio, desde una perspectiva global, teniendo en cuenta dos aspectos: el primero de ellos

Rev. Diálogo Educ., Curitiba, v. 8, n. 25, p. 633-645, set./dez. 2008 
es que la reforma supuso una práctica didáctica que se concretó en la elaboración de un currículo y en la creación de nuevos medios pedagógicos; el segundo, que esta práctica docente se fundamenta en un cuadro ideológico que la condiciona y que va a ser determinante en la tarea emprendida; este cuadro ideológico no es otro que el estructuralismo bourbakista.

Se han considerado los siguientes aspectos:

- análisis crítico del método axiomático y estructuras;

- conocimiento "común" y Matemáticas "modernas";

- análisis crítico de la representación de conjuntos mediante diagramas de Venn;

- aspectos críticos del lenguaje de las flechas;

- geometría vectorial versus geometría euclídea.

En resumen, como resultado de este estudio crítico, se ha llegado a que:

I. La puesta en práctica de la concepción bourbakista trajo como consecuencia olvidar otros aspectos del pensamiento matemático. Bouvier (1981), entre otros, ha señalado el poder motriz de las conjeturas en la actividad matemática. Sin embargo, las conjeturas, tanteos, pruebas y refutaciones, lo que Lakatos llamó "matemática informal" no tiene cabida en la enseñanza del CBPM;

II. En el proceso de hacer explícito lo implícito, se observa que en la experiencia llevada a cabo, sobre todo en la enseñanza primaria, Fréderique Papy y colaboradores reconocen, a veces, un cierto índice de conocimiento matemático en los alumnos aunque esto está motivado por hechos banales; se da, en cierto modo, lo que Brousseau (1986) ha llamado el efecto "Jourdain";

III. En lo que se refiere a la representación de conjuntos mediante diagramas de Venn, se llega a situaciones extrañas; por ejemplo, representar un león por un punto dentro de la cuerda del diagrama que rodea el conjunto de los animales, y la nariz del león por un punto fuera de dicha curva cerrada. Freudenthal (1973) será extremadamente crítico con este tipo de situaciones.Por su parte Brousseau (1986) aludirá explícitamente a la metodología de Papy, considerando que muchas veces el profesor puede llega a tomar sus métodos heurísticos como objetos propios de estudio en lugar del verdadero conocimiento matemático. Brousseau denominará este efecto como "deslizamiento metacognitivo";

Rev. Diálogo Educ., Curitiba, v. 8, n. 25, p. 633-645, set./dez. 2008 
IV. En lo que se refiere al "lenguaje de las flechas" a medida que avanza la experiencia los grafos multicolores o papygramas se van utilizando cada vez más; así, en cuarto curso de primaria su uso será tan generalizado que prácticamente para cualquier cuestión que se plantea se utilizan grafos. De este modo el lenguaje de las flechas pasa de ser un medio a convertirse en un fin; además, analizando la experiencia se llega a la conclusión de que dicho lenguaje, llevado hasta puntos tan extremos, es equívoco. Se produce, en definitiva, una recreación intelectual de los autores se la experiencia en los medios utilizados, que dejan de serlo para convertirse, a su vez, en objetos de enseñanza;

V. En cuanto a la supresión de la enseñanza de la geometría sustituida por el álgebra se ha hecho una confrontación de las ideas de Atiyah (1976; 1982), R. Thom (1971; 1973), Freudenthal (1973) con las de Dieudonné (1973; 1976) estas últimas asumidas por Papy. En el fondo de esta polémica subyace una cuestión epistemológica. Dieudonné y Papy no captan el sentido del debate sobre el papel a asignar a la geometría en la enseñanza, sencillamente porque desde su punto de vista dicho problema no existe. Papy está plenamente convencido de que está enseñando geometría porque identifica la geometría con el álgebra lineal en concordancia con la idea de "álgebra geométrica" de Artin (1957), que es fuente de inspiración de su renovación de la enseñanza de las Matemáticas.

\section{REFERÊNCIAS}

ARTIN, E. Geometric algebra. New York: Interscience Publishers, 1957.

ATIYAH, M. Trends in pure mathematics. In: INTERNATIONAL CONGRESS ON MATHEMATICAL EDUCATION, 3., 1976, Karlsruhe. Proceedings... Karlsruhe: ZDM, 1976. p. 61-74.

ATIYAH, M. What is geometry? The Mathematical Gazzette, v. 66, n. 437 , p. $179-184$, Oct. 1982.

BOUVIER, A. La mystification mathématique. Paris: Hermann, 1981.

BROUSSEAU, G. Theorisations des phénomenés d'enseignemet des mathématiques. 1986. $481 \mathrm{f}$. Tesis (Docteur d'E'tat ès Sciencies) - Université de Bordeaux I, Bordeaux, 1986.

Rev. Diálogo Educ., Curitiba, v. 8, n. 25, p. 633-645, set./dez. 2008 
CASTELNUOVO, E. Didáctica de la matemática moderna. México: Trillas, 1963.

DIEUDONNÉ, J. Should we teach "Modern" mathematics? American Scientist, v. 61, n. 1, p. 16-19, 1973.

DIEUDONNÉ, J. L'abstraction et l'intuition mathématique. NICO, n. 20, p. 45-66, 1976.

FREUDENTHAL, H. Mathematics as an educational task. Dordrecht: D. Reidel, 1973.

HILBERT, D. Grundlagen der Geometrie. Stuttgart: B. G. Teubner Verlag, 1899.

HOWSON, G. Análisis crítico del desarrollo curricular en matemáticas. In: STEINER H; CHRISTIANSEN, B. (Ed.). Nuevas tendencias en la enseñanza de las matemáticas. Paris: Unesco, 1979. v. 4, p. 151-183.

HOWSON, G.; KEITEL, C.; KILPATRICK, J. Currículum development in mathematics. Cambridge: Cambridge University Press, 1981.

SIERRA, M. La reforma de la enseñanza de las matemáticas después de la Segunda Guerra Mundial: aportación del centre belge de pédagogie de la mathématique (CBPM). 1989. 391 f. Tesis (Doctorado en Educación) - Universidad de Salamanca, Salamanca, 1989.

THOM, R. Modern mathematics: an educational and philosophic error? American Scientist, v. 59, n. 6, p. 695-699, Nov. 1971.

THOM, R. Modern mathematics: does it exist? In: HOWSON, A. G. (Ed.). Developments in mathematical education. Cambridge: Cambridge University Press, 1973. p. 194-209.

Recebido: 26/05/2008

Recebido: 05/26/2008

Aprovado: 01/07/2008

Aprobado: 07/01/2008

Rev. Diálogo Educ., Curitiba, v. 8, n. 25, p. 633-645, set./dez. 2008 